\title{
Correlated Electron-Nuclear Dynamics with Conditional Wave Functions
}

\author{
Guillermo Albareda, ${ }^{1, *}$ Heiko Appel, ${ }^{1}$ Ignacio Franco, ${ }^{2}$ Ali Abedi, ${ }^{3}$ and Angel Rubio ${ }^{1,4,}$ \\ ${ }^{1}$ Fritz-Haber-Institut der Max-Planck-Gesellschaft, Faradayweg 4-6, D-14195 Berlin, Germany \\ ${ }^{2}$ Department of Chemistry, University of Rochester, Rochester, New York 14627, USA \\ ${ }^{3}$ Max-Planck Institut für Mikrostrukturphysik, Weinberg 2, D-06120 Halle, Germany \\ ${ }^{4}$ Nano-Bio Spectroscopy Group and ETSF Scientific Development Centre, Universidad del País Vasco, \\ CFM CSIC-UPV/EHU-MPC and DIPC, Avenida Tolosa 72, E-20018 Donostia, Spain
}

(Received 21 May 2014; published 21 August 2014)

\begin{abstract}
The molecular Schrödinger equation is rewritten in terms of nonunitary equations of motion for the nuclei (or electrons) that depend parametrically on the configuration of an ensemble of generally defined electronic (or nuclear) trajectories. This scheme is exact and does not rely on the tracing out of degrees of freedom. Hence, the use of trajectory-based statistical techniques can be exploited to circumvent the calculation of the computationally demanding Born-Oppenheimer potential-energy surfaces and nonadiabatic coupling elements. The concept of the potential-energy surface is restored by establishing a formal connection with the exact factorization of the full wave function. This connection is used to gain insight from a simplified form of the exact propagation scheme.
\end{abstract}

DOI: 10.1103/PhysRevLett.113.083003

PACS numbers: 31.15.X-, 31.15.A-, 31.50.-x

In order to describe the correlated motion of electrons and nuclei, many strategies have been proposed to transcend the picture where the nuclei evolve on top of a single Born-Oppenheimer potential-energy surface (BOPES) [1]. Using a time-independent basis-set expansion of the electron-nuclear wave function, full quantum studies provide a complete description of nonadiabatic dynamics [2]. The scaling of these methods (even for a time-dependent basis-set expansion [3]) is, however, limiting their use to describe a few degrees of freedom. The so-called direct dynamics techniques attempt to alleviate this problem by calculating the BOPESs on the fly [4]. Of particular interest here are those methods that use information from quantum chemistry or time-dependent density functional theory calculations in the form of forces. Ab initio surface hopping, Ehrenfest dynamics [5], or Gaussian wave packet methods (such as the multiple spawning method) [6] are all able to reproduce the dynamics of some systems of interest [7]. In most of these methods, however, the form of the nuclear wave function is restricted, as they use a local or classical trajectory-based representation of the nuclear wave packet. In addition to the difficulties of including external fields or calculating the nonadiabatic coupling elements (NACs), this introduces the problem of systematically accounting for quantum nuclear effects.

In this Letter, we propose an exact propagation scheme aimed at the study of nonadiabatic dynamics in the presence of arbitrary external electromagnetic fields. The coupled electron-nuclear dynamics is separated without tracing out degrees of freedom, which lends itself to a rigorous starting point for systematically including nonadiabatic nuclear effects without relying on the computation of BOPESs and NACs. This work constitutes a multicomponent extension of the conditional formalism proposed in Refs. [8,9]. Further, the propagation scheme presented here generalizes the conditional formalism beyond its original hydrodynamic formulation [8]. This makes it suitable to be coupled with well established electronic structure methods.

Throughout this Letter, we use atomic units, and electronic and nuclear coordinates are collectively denoted by $\mathbf{r}=\left\{\mathbf{r}_{1}, \ldots, \mathbf{r}_{N_{e}}\right\}$ and $\mathbf{R}=\left\{\mathbf{R}_{1}, \ldots, \mathbf{R}_{N_{n}}\right\}$, with $N_{e}$ and $N_{n}$, respectively, being the total numbers of electrons and nuclei. The full (nonrelativistic) electron-nuclear wave function $\Psi(\mathbf{r}, \mathbf{R}, t)$ satisfies the time-dependent Schrödinger equation (TDSE)

$$
i \partial_{t} \Psi(t)=\left\{\hat{T}_{e}(\mathbf{r})+\hat{T}_{n}(\mathbf{R})+\hat{W}(\mathbf{r}, \mathbf{R}, t)\right\} \Psi(t),
$$

where $\hat{T}_{e}=\sum_{\xi=1}^{N_{e}}\left(-i \nabla_{\xi}-\mathbf{A}^{\mathcal{E M}}\left(\mathbf{r}_{\xi}\right)\right)^{2} / 2 m$ and $\hat{T}_{n}=$ $\sum_{\nu=1}^{N_{n}}\left(-i \nabla_{\nu}-\mathbf{A}^{\mathcal{E} \mathcal{M}}\left(\mathbf{R}_{\nu}\right)\right)^{2} / 2 M_{\nu}$ are the electronic and nuclear kinetic energy operators, and $\mathbf{A}^{\mathcal{E M}}$ is the external vector potential in the Coulomb gauge due to an arbitrary external electromagnetic field. All scalar potentials are included in $\hat{W}(\mathbf{r}, \mathbf{R}, t)=\hat{V}_{\text {int }}(\mathbf{r}, \mathbf{R})+V_{\mathrm{ext}}^{e}(\mathbf{r}, t)+\hat{V}_{\mathrm{ext}}^{n}$ $(\mathbf{R}, t)$, where $V_{\mathrm{ext}}^{e}\left(V_{\mathrm{ext}}^{n}\right)$ is the electronic (nuclear) external scalar potential, and $\hat{V}_{\text {int }}=\hat{W}_{e e}(\mathbf{r})+\hat{W}_{n n}(\mathbf{R})+\hat{W}_{e n}(\mathbf{r}, \mathbf{R})$ accounts for the internal Coulombic interactions. Next, we present the main result of this Letter.

Theorem.- (a) The molecular wave function $\Psi(\mathbf{r}, \mathbf{R}, t)$ satisfying the TDSE (1) can be exactly decomposed either in terms of nuclear or electronic conditional wave functions

$$
\begin{gathered}
\psi_{n}\left(\mathbf{R}, t ; \mathbf{r}^{\alpha}(t)\right):=\int \delta\left(\mathbf{r}^{\alpha}(t)-\mathbf{r}\right) \Psi(\mathbf{r}, \mathbf{R}, t) d \mathbf{r}, \\
\psi_{e}\left(\mathbf{r}, t ; \mathbf{R}^{\alpha}(t)\right):=\int \delta\left(\mathbf{R}^{\alpha}(t)-\mathbf{R}\right) \Psi(\mathbf{r}, \mathbf{R}, t) d \mathbf{R},
\end{gathered}
$$


provided that the ensemble of trajectories $\left\{\mathbf{r}^{\alpha}(t), \mathbf{R}^{\alpha}(t)\right\}$ explores the support of $|\Psi(\mathbf{r}, \mathbf{R}, t)|^{2}$ at any time $t$.

(b) The conditional wave functions $\psi_{n}\left(\mathbf{R}, t ; \mathbf{r}^{\alpha}(t)\right)$ and $\psi_{e}\left(\mathbf{r}, t ; \mathbf{R}^{\alpha}(t)\right)$ obey, respectively, the following nonunitary equations of motion:

$$
\begin{aligned}
i d_{t} \psi_{n}\left(\mathbf{R}, t ; \mathbf{r}^{\alpha}(t)\right)= & \left\{\hat{T}_{n}+\hat{W}\left(\mathbf{r}^{\alpha}(t), \mathbf{R}, t\right)\right\} \psi_{n}\left(\mathbf{R}, t ; \mathbf{r}^{\alpha}(t)\right) \\
& +\left.\hat{T}_{e} \Psi(\mathbf{r}, \mathbf{R}, t)\right|_{\mathbf{r}^{\alpha}(t)} \\
& +\left.i \nabla_{\mathbf{r}} \Psi(\mathbf{r}, \mathbf{R}, t)\right|_{\mathbf{r}^{\alpha}(t)} \cdot \dot{\mathbf{r}}^{\alpha}(t), \\
i d_{t} \psi_{e}\left(\mathbf{r}, t ; \mathbf{R}^{\alpha}(t)\right)= & \left\{\hat{T}_{e}+\hat{W}\left(\mathbf{r}, \mathbf{R}^{\alpha}(t), t\right)\right\} \psi_{e}\left(\mathbf{r}, t ; \mathbf{R}^{\alpha}(t)\right) \\
& +\left.\hat{T}_{n} \Psi(\mathbf{r}, \mathbf{R}, t)\right|_{\mathbf{R}^{\alpha}(t)} \\
& +\left.i \nabla_{\mathbf{R}} \Psi(\mathbf{r}, \mathbf{R}, t)\right|_{\mathbf{R}^{\alpha}(t)} \cdot \dot{\mathbf{R}}^{\alpha}(t) .
\end{aligned}
$$

For the sake of simplicity, we omit from now on the explicit time dependence of the trajectories, i.e., $\left\{\mathbf{r}^{\alpha}, \mathbf{R}^{\alpha}\right\} \equiv$ $\left\{\mathbf{r}^{\alpha}(t), \mathbf{R}^{\alpha}(t)\right\}$.

Proof.-(a) To demonstrate that expressions (2) and (3) are exact decompositions of the molecular wave function, we only need to realize that an ensemble of these conditional wave functions can be used to reconstruct the full wave function as follows:

$$
\Psi(\mathbf{r}, \mathbf{R}, t)=\left\{\begin{array}{cc}
\hat{\mathcal{D}}_{\mathbf{r}}\left[\psi_{n}\right] & \text { if } \sum_{\alpha=1}^{\infty} \delta\left(\mathbf{r}^{\alpha}-\mathbf{r}\right) \neq 0 \\
0 & \text { if } \sum_{\alpha=1}^{\infty} \delta\left(\mathbf{r}^{\alpha}-\mathbf{r}\right)=0
\end{array}\right.
$$

or

$$
\Psi(\mathbf{r}, \mathbf{R}, t)=\left\{\begin{array}{cc}
\hat{\mathcal{D}}_{\mathbf{R}}\left[\psi_{e}\right] & \text { if } \sum_{\alpha=1}^{\infty} \delta\left(\mathbf{R}^{\alpha}-\mathbf{R}\right) \neq 0 \\
0 & \text { if } \sum_{\alpha=1}^{\infty} \delta\left(\mathbf{R}^{\alpha}-\mathbf{R}\right)=0,
\end{array}\right.
$$

where we have defined the transformations $\hat{\mathcal{D}}_{\mathbf{r}}\left[f\left(\mathbf{r}^{\alpha}\right)\right] \equiv$ $\sum_{\alpha=1}^{\infty} \delta\left(\mathbf{r}^{\alpha}-\mathbf{r}\right) f\left(\mathbf{r}^{\alpha}\right) / \sum_{\alpha=1}^{\infty} \delta\left(\mathbf{r}^{\alpha}-\mathbf{r}\right) \quad$ and $\quad \hat{\mathcal{D}}_{\mathbf{R}}\left[g\left(\mathbf{R}^{\alpha}\right)\right] \equiv$ $\sum_{\alpha=1}^{\infty} \delta\left(\mathbf{R}^{\alpha}-\mathbf{R}\right) g\left(\mathbf{R}^{\alpha}\right) / \sum_{\alpha=1}^{\infty} \delta\left(\mathbf{R}^{\alpha}-\mathbf{R}\right)$, connecting, respectively, the (parametrized) electronic and nuclear subspaces with the full configuration space. Introducing the definitions (2) and (3), respectively, into Eqs. (6) and (7), the full wave function $\Psi(\mathbf{r}, \mathbf{R}, t)$ is immediately recovered, provided that $\left\{\mathbf{r}^{\alpha}, \mathbf{R}^{\alpha}\right\}$ exhaust the support of $|\Psi(\mathbf{r}, \mathbf{R}, t)|^{2}$. Notice that the second condition in Eqs. (6) and (7) is required in order to avoid singularities due to the formation of nodes.

(b) Equations (4) and (5) can be derived by evaluating the Schrödinger equation (1) at the configuration of the electronic and nuclear trajectories, respectively, $\mathbf{r}^{\alpha}$ and $\mathbf{R}^{\alpha}$ and using the chain rule to write the time derivatives as $d_{t} \psi_{n}=\partial_{t} \psi_{n}+\left.\nabla_{\mathbf{r}} \Psi\right|_{\mathbf{r}^{\alpha}} \cdot \dot{\mathbf{r}}^{\alpha}$ and $d_{t} \psi_{e}=\partial_{t} \psi_{e}+\left.\nabla_{\mathbf{R}} \Psi\right|_{\mathbf{R}^{\alpha}} \cdot \dot{\mathbf{R}}^{\alpha}$.

As written in Eqs. (2) and (3), $\psi_{n}\left(\mathbf{R}, t ; \mathbf{r}^{\alpha}\right)$ and $\psi_{e}\left(\mathbf{r}, t ; \mathbf{R}^{\alpha}\right)$ represent $3 N_{n^{-}}$and $3 N_{e^{-}}$-dimensional slices of the full molecular wave function taken along the nuclear and electronic coordinates, respectively. Each conditional wave function constitutes, in this regard, an open quantum system. Their evolution is nonunitary due to the last two terms in Eqs. (4) and (5), in general, complex functionals of the full wave function. More specifically, the first complex functional in Eqs. (4) and (5) describes the interaction among conditional wave functions of the same species, while the last term represents the advection of nuclear (electronic) conditional wave functions along the electronic (nuclear) trajectories. In the most general case, both advective and kinetic correlation terms are required to preserve the norm of the full wave function and to conserve the total energy.

The nonunitarity of Eqs. (4) and (5) is the result of separating a certain number of degrees of freedom without tracing over the rest. From this point of view, the propagation of the nuclear equations of motion (4) does not require the calculation of BOPESs or NACs. This makes the method particularly advantageous when studying processes that involve many BOPESs or external electromagnetic fields, as in laser-induced dynamics or scattering from metallic surfaces.

Let us emphasize that the decomposition of the molecular wave function in Eqs. (2) and (3) is only one case among many other possible conditional decompositions. The above theorem provides a general prescription to decompose the electron-nuclear wave function into a complete set of conditional wave functions. Particularly appealing is also the separation of the full wave function into single-particle conditional wave functions, i.e., $\psi_{n}^{\nu}\left(\mathbf{R}_{\nu}, t ; \mathbf{R}_{1}^{\alpha}, \ldots, \mathbf{R}_{\nu-1}^{\alpha}\right.$, $\left.\mathbf{R}_{\nu+1}^{\alpha}, \ldots, \mathbf{r}^{\alpha}\right)$ and $\psi_{e}^{\xi}\left(\mathbf{r}_{\xi}, t ; \mathbf{r}_{1}^{\alpha}, \ldots, \mathbf{r}_{\xi-1}^{\alpha}, \mathbf{r}_{\xi+1}^{\alpha}, \ldots, \mathbf{R}^{\alpha}\right)$. Since the initial conditions of a trajectory-based simulation can be generated with importance-sampling techniques, conditional decompositions allow us to circumvent the problem of storing and propagating a many-particle wave function whose size scales exponentially with the number of particles.

In the above theorem, it remains to specify the trajectories $\left\{\mathbf{r}^{\alpha}, \mathbf{R}^{\alpha}\right\}$. As already mentioned, the only requirement to be fulfilled by these trajectories is that they must explore the support of the quantum-probability density $|\Psi(\mathbf{r}, \mathbf{R}, t)|^{2}$. Notice that for the simplest case, where $\dot{\mathbf{r}}^{\alpha}=\dot{\mathbf{R}}^{\alpha}=0$, Eqs. (4) and (5) both reduce to the TDSE (1). Alternatively, other choices of $\left\{\mathbf{r}^{\alpha}, \mathbf{R}^{\alpha}\right\}$ can be used to circumvent the use of computationally demanding fixedgrid methods. Here, we choose $\left\{\mathbf{r}^{\alpha}, \mathbf{R}^{\alpha}\right\}$ to be Bohmian trajectories because they do sample the quantum-probability density [10] and because they provide, in addition, an intuitive picture of quantum dynamics [11]. Specifically, a proper sampling of the initial electron-nuclear wave function guarantees that $|\Psi(\mathbf{r}, \mathbf{R}, t)|^{2}$ is exactly reproduced at any time by quantum trajectories $\left\{\mathbf{r}^{\alpha}, \mathbf{R}^{\alpha}\right\}$ defined as

$$
\begin{array}{r}
\mathbf{r}_{\xi}^{\alpha}(t)=\mathbf{r}_{\xi}^{\alpha}\left(t_{0}\right)+\int_{t_{0}}^{t} \mathbf{v}_{\xi}^{e}\left(\mathbf{r}^{\alpha}\left(t^{\prime}\right), \mathbf{R}^{\alpha}\left(t^{\prime}\right), t^{\prime}\right) d t^{\prime}, \\
\mathbf{R}_{\nu}^{\alpha}(t)=\mathbf{R}_{\nu}^{\alpha}\left(t_{0}\right)+\int_{t_{0}}^{t} \mathbf{v}_{\nu}^{n}\left(\mathbf{r}^{\alpha}\left(t^{\prime}\right), \mathbf{R}^{\alpha}\left(t^{\prime}\right), t^{\prime}\right) d t^{\prime},
\end{array}
$$


where electronic and nuclear velocity fields are defined as $\quad \mathbf{v}_{\xi}^{e}(\mathbf{r}, \mathbf{R}, t)=\left(\nabla_{\xi} S-\mathbf{A}^{\mathcal{E M}}\right) / m \quad$ and $\quad \mathbf{v}_{\nu}^{n}(\mathbf{r}, \mathbf{R}, t)=$ $\left(\nabla_{\nu} S-\mathbf{A}^{\mathcal{E M}}\right) / M_{\nu}$, and $S(\mathbf{r}, \mathbf{R}, t)$ is the phase of the full wave function $\Psi=|\Psi| e^{i S}[10]$. Note that the choice of Bohmian trajectories is not mandatory. Alternatively, trajectory-based Monte Carlo or importance-sampling techniques can be used, provided that they sample the quantum-probability density.

While not required in principle, in practice, it is useful to propagate both the nuclear and electronic conditional wave functions [Eqs. (4) and (5)] to compute the quantum trajectories via conditional velocity fields defined as $\mathbf{v}_{\xi}^{e}\left(\mathbf{r}^{\alpha}, t ; \mathbf{R}^{\alpha}\right)=$ $\left(\nabla_{\xi} S_{e}\left(\mathbf{r}, t ; \mathbf{R}^{\alpha}\right)-\mathbf{A}^{\mathcal{E M}}\left(\mathbf{r}_{\xi}\right)\right) /\left.m\right|_{\mathbf{r}^{\alpha}} \quad$ and $\quad \mathbf{v}_{\nu}^{n}\left(\mathbf{R}^{\alpha}, t ; \mathbf{r}^{\alpha}\right)=$ $\left(\nabla_{\nu} S_{n}\left(\mathbf{R}, t ; \mathbf{r}^{\alpha}\right)-\mathbf{A}^{\mathcal{E} \mathcal{M}}\left(\mathbf{R}_{\nu}\right)\right) /\left.M_{\nu}\right|_{\mathbf{R}^{\alpha}}$, where $S_{e}\left(\mathbf{r}, t ; \mathbf{R}^{\alpha}\right)$ and $S_{n}\left(\mathbf{R}, t ; \mathbf{r}^{\alpha}\right)$ are, respectively, the phases of the electronic and nuclear conditional wave functions $\psi_{e}=\left|\psi_{e}\right| e^{i S_{e}}$ and $\psi_{n}=\left|\psi_{n}\right| e^{i S_{n}}$. In this way, the reconstruction of the full wave function is avoided at the expense of solving twice the number of equations of motion $[8,9]$.

In the remaining part of the Letter, we explore a first approximation to this general method to solve the vibronic problem. Let us first consider the external vector potential $\mathbf{A}^{\mathcal{E M}}$ to be 0 . In addition, we assume a zero order expansion of the complex functionals in Eqs. (4) and (5) around the nuclear and electronic variables, respectively, i.e., $\left.\hat{T}_{e} \Psi\right|_{\mathbf{r}^{\alpha}}+\left.i \nabla_{\mathbf{r}} \Psi\right|_{\mathbf{r}^{\alpha}} \cdot \dot{\mathbf{r}}^{\alpha}=f_{n}\left(\mathbf{r}^{\alpha}, t\right)$ and $\left.\hat{T}_{n} \Psi\right|_{\mathbf{R}^{\alpha}}+$ $\left.i \nabla_{\mathbf{R}} \Psi\right|_{\mathbf{R}^{\alpha}} \cdot \dot{\mathbf{R}}^{\alpha}=f_{e}\left(\mathbf{R}^{\alpha}, t\right)$. Notice that this approximation corresponds to a Hermitian limit of Eqs. (4) and (5) and thus that the time evolution of $\psi_{n}\left(\mathbf{R}, t ; \mathbf{r}^{\alpha}\right)$ and $\psi_{e}\left(\mathbf{r}, t ; \mathbf{R}^{\alpha}\right)$ becomes unitary. The approximated functionals entail now a pure time-dependent phase that can be omitted because the velocity fields $v_{\nu}^{n}(\mathbf{r}, \mathbf{R}, t)$ and $v_{\xi}^{e}(\mathbf{r}, \mathbf{R}, t)$ are invariant under such a global phase transformation. We call the resulting propagation scheme, i.e., Eqs. (8) and (9), together with the Hermitian limit of Eqs. (4) and (5) as described above, the Hermitian conditional approach. Remarkably, this scheme does not require the computation of the quantum potential, in this manner overcoming a common bottleneck in quantum trajectory-based approaches [12].

To assess this approximated scheme, it is useful to restore the concept of the potential-energy surface. This can be done by connecting this general method to the exact factorization of the molecular wave function [13]. By rewriting the nuclear conditional wave function as a direct product of electronic and nuclear probability amplitudes, i.e.,

$$
\psi_{n}\left(\mathbf{R}, t ; \mathbf{r}^{\alpha}\right)=\Phi_{\mathbf{R}}\left(\mathbf{r}^{\alpha}, t\right) \chi(\mathbf{R}, t),
$$

equations of motion for both terms in Eq. (10) can be derived. Of particular interest is here $\chi(\mathbf{R}, t)$ because it allows us to isolate the role played by each term in Eq. (4) on the dynamics of the nuclear probability density in terms of a time-dependent potential-energy surface [14].
In particular, the Hermitian limit of Eq. (4) leads to the following equation of motion for $\chi(\mathbf{R}, t)$ [14]:

$$
\begin{aligned}
i \partial_{t} \chi(\mathbf{R}, t)=\{ & \sum_{\nu=1}^{N_{n}} \frac{1}{2 M_{\nu}}\left(-i \nabla_{\nu}+\mathcal{A}_{\nu}(\mathbf{R}, t)\right)^{2} \\
& \left.+\hat{V}_{\mathrm{ext}}^{n}(\mathbf{R}, t)+\tilde{\epsilon}(\mathbf{R}, t)\right\} \chi(\mathbf{R}, t),
\end{aligned}
$$

where $\mathcal{A}_{\nu}(\mathbf{R}, t)$ is the $\nu$ component of the time-dependent Berry phase [13], and the approximated time-dependent potential-energy surfaces $\tilde{\epsilon}(\mathbf{R}, t)$ are defined as

$$
\begin{gathered}
\tilde{\epsilon}(\mathbf{R}, t)=\epsilon(\mathbf{R}, t) \\
-\int \hat{\mathcal{D}}_{\mathbf{r}}\left[\left.\Phi_{\mathbf{R}}^{*}\left(\mathbf{r}^{\alpha}, t\right)\left(\left(\hat{T}_{e}+\dot{\mathbf{r}}^{\alpha} \cdot \nabla_{\mathbf{r}}\right) \Phi_{\mathbf{R}}(\mathbf{r}, t)\right)\right|_{\mathbf{r}^{\alpha}}\right] d \mathbf{r},
\end{gathered}
$$

where $\Phi_{\mathbf{R}}\left(\mathbf{r}^{\alpha}, t\right)=\psi_{n}\left(\mathbf{R}, t ; \mathbf{r}^{\alpha}\right) / \chi(\mathbf{R}, t)$. Equation (11) establishes a direct correspondence between the complex functionals in Eq. (4) and the last term on the rhs of Eq. (12). Note that computing the nuclear probability density from such approximated nuclear conditional wave functions as $|\chi(\mathbf{R}, t)|^{2}=\int d \mathbf{r} \hat{\mathcal{D}}_{\mathbf{r}}\left[\left|\psi_{n}\left(\mathbf{R}, t ; \mathbf{r}^{\alpha}\right)\right|^{2}\right]$ is equivalent to propagating the nuclear probability density according to Eqs. (11) and (12) [14]. Neglecting the averaged electronic kinetic energy in Eq. (12) could seem a crude approximation in the Born-Oppenheimer limit [13]; however, in the following example we show that this is not the case when nonadiabatic effects are important.

In what follows, we address two distinctive aspects of the correlated electron-nuclear motion, namely, tunneling and interferences. A detailed discussion of the performance of the Hermitian conditional scheme to describe the splitting of the nuclear probability density can be found in Ref. [15]. A numerically exactly solvable problem that exhibits the characteristic features associated with nonadiabatic processes is the model of Shin and Metiu [18], which consists of three ions and a single electron. Two ions are fixed at a distance $L=19.0 a_{0}$, and the third ion and the electron are free to move in one dimension along the line joining the fixed ions. The Hamiltonian for this system reads

$$
\begin{aligned}
\hat{H}(r, R)= & -\frac{1}{2} \frac{\partial^{2}}{\partial r^{2}}-\frac{1}{2 M} \frac{\partial^{2}}{\partial R^{2}}+\frac{1}{\left|\frac{L}{2}-R\right|}+\frac{1}{\left|\frac{L}{2}+R\right|} \\
& -\frac{\operatorname{erf}\left(\frac{|R-r|}{R_{f}}\right)}{|R-r|}-\frac{\operatorname{erf}\left(\frac{|r-L / 2|}{R_{r}}\right)}{\left|r-\frac{L}{2}\right|}-\frac{\operatorname{erf}\left(\frac{|r+L / 2|}{R_{l}}\right)}{\left|r+\frac{L}{2}\right|},
\end{aligned}
$$

where the symbols $\mathbf{r}$ and $\mathbf{R}$ are replaced by $r$ and $R$, and the coordinates of the electron and the movable nucleus are measured from the center of the two fixed ions. For the remaining parameters, we choose $M=1836$ a.u. and $R_{f}=7 a_{0}, R_{l}=4.4 a_{0}$, and $R_{r}=3.1 a_{0}$, such that the first BOPES $\epsilon_{\mathrm{BO}}^{(1)}$ is strongly coupled to the second BOPES $\epsilon_{\mathrm{BO}}^{(2)}$ 


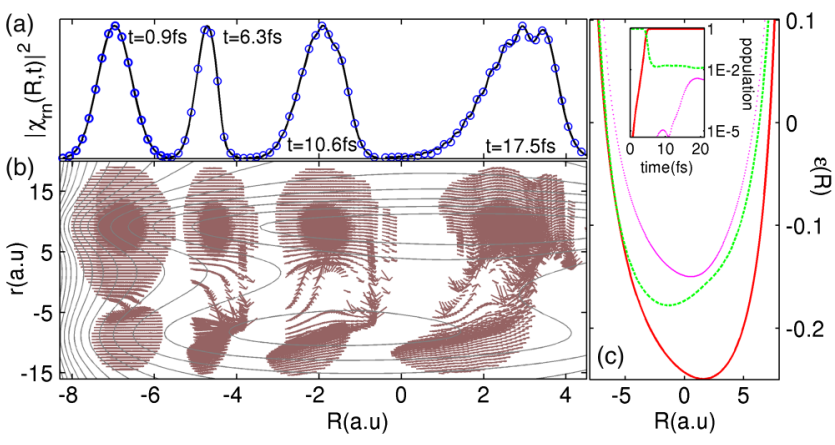

FIG. 1 (color online). (a) Exact (solid black line) and approximated (blue circles) nuclear probability densities renormalized as $\left|\chi_{\mathrm{rn}}(\mathbf{R}, t)\right|^{2}=|\chi(\mathbf{R}, t)|^{2} / \max |\chi(\mathbf{R}, t)|^{2}$ at four different times. (b) Arrows refer to the (two-dimensional) velocity field computed from the approximated conditional wave functions. The gray contour lines represent the corresponding electron-nuclear twodimensional potential-energy surface. (c) First (red line), second (green line), and third (magenta line) BOPESs involved in the nonadiabatic process. Inset: Adiabatic populations as a function of time computed from the exact solution.

within an extended region defined by $R<-4 a_{0}$. In addition, there is a moderate coupling between the second BOPES $\epsilon_{\mathrm{BO}}^{(2)}$ and the third BOPES $\epsilon_{\mathrm{BO}}^{(3)}$ for $R>2 a_{0}$ [see Fig. 1(c)]. The coupling to the rest of the BOPESs is negligible. We suppose the system to be initially excited to $\epsilon_{\mathrm{BO}}^{(2)}$ and the initial nuclear wave function to be a Gaussian wave packet with $\sigma=1 / \sqrt{2.85}$, centered at $R=-7.0 a_{0}$; i.e., the initial full wave function is $\Psi\left(r, R, t_{0}\right)=$ $A e^{-(R+7)^{2} / \sigma^{2}} \Phi_{R}^{(2)}(r)$, with $A$ being a normalization constant. Starting with $\Psi\left(r, R, t_{0}\right)$, we first sample its probability density with trajectories and then propagate Eqs. (8) and (9) together with the Hermitian limit of Eqs. (4) and (5). In Fig. 1(a), we show snapshots at different times of the nuclear probability density for the exact calculation (solid black line) and for the approximated solution (blue circles) computed as $|\chi(\mathbf{R}, t)|^{2}=\int d \mathbf{r} \hat{\mathcal{D}}_{\mathbf{r}}\left[\left|\psi_{n}\left(\mathbf{R}, t ; \mathbf{r}^{\alpha}\right)\right|^{2}\right]$. Showing an excellent agreement, this propagation scheme is demonstrated to capture not only the conspicuous electronic transition between $\epsilon_{\mathrm{BO}}^{(2)}$ and $\epsilon_{\mathrm{BO}}^{(1)}$ but also the interferences originating at later times from contributions of higher adiabatic populations [see the rise of the population of $\epsilon_{\mathrm{BO}}^{(3)}$ in the inset of Fig. 1(c)]. The accuracy of the Hermitian scheme for this particular example relies on the fact that the first complex functionals in Eqs. (4) and (5) are irrelevant, as they become almost constant along the nuclear and electronic coordinates, respectively. In addition, the advective terms are negligible within the support of the electronnuclear wave function [19].

The BOPESs constitute a formidable interpretative tool to understand the electron-nuclear coupled dynamics; however, they provide here a biased picture of the dynamics guiding the transit from the initial state at $t=0$ fs to the final state at $t=17.5 \mathrm{fs}$. Alternatively, here, we gain insight into this dynamics by analyzing the quantum velocity fields $\mathbf{v}^{n}(r, R, t)$ and $\mathbf{v}^{e}(r, R, t)$ computed, respectively, from the approximated conditional wave functions $\psi_{n}\left(\mathbf{R}, t ; \mathbf{r}^{\alpha}\right)$ and $\psi_{e}\left(\mathbf{r}, t ; \mathbf{R}^{\alpha}\right)$. Snapshots of these velocity fields in terms of arrow maps are displayed in Fig. 1(b) together with contour lines representing the two-dimensional potential-energy surface. The first thing to notice is the fact that while in the picture of the BOPESs, initial and final states are connected via tunneling (along the nuclear coordinates), no tunneling is indeed taking place along this direction in the configuration space. At $t=0.9 \mathrm{fs}$, the trajectories at the rear of the wave packet (with respect to the nuclear coordinates) carry a large momentum that forces the molecular wave function to squeeze at later times (e.g., at $t=6.3 \mathrm{fs}$ ). This contraction in the nuclear coordinates is accompanied by a stretching of the wave function in the electronic coordinates that leads to a dripping of probability density out of the main "reaction path" via tunneling. During the tunneling process, quantum trajectories undergo a very fast motion in the electronic direction [notice the different sizes of the arrows in Fig. 1(b)]. This induces a (tunneling) back and forth flow of probability density from one valley to the other (see the snapshots at times $t=10.6 \mathrm{fs}$ and $t=17.5 \mathrm{fs}$ ). As a direct consequence, an interference pattern originates in close analogy with the quantum "bobsled effect" described by McCullough and Wyatt [20]. A remarkably vortical behavior, reminiscent of the quantum "whirlpool effect" [20], can be also observed when quasinodes in the full wave function develop at $t=10.6 \mathrm{fs}$ and $t=17.5 \mathrm{fs}$. This example demonstrates that the conditional formalism, even in the Hermitian limit, provides a powerful tool to describe complex features ubiquitous in nonadiabatic processes such as tunneling or interferences. Further, it evidences the interpretative value of the conditional formalism to grasp the "microscopic" behavior of quantum dynamics in terms of local velocities.

To summarize, we present an exact trajectory-based decomposition of the Schrödinger equation in terms of conditional nuclear and electronic wave functions (2) and (3). Their evolution according to Eqs. (4) and (5) is nonunitary and lends itself as a rigorous procedure to tackle open quantum systems by means of trajectory-based statistical techniques. In particular, the propagation of Eq. (4) does not entail integrals over the electronic degrees of freedom, and hence we expect it to be of particular interest in scenarios where several BOPESs and external electromagnetic fields are involved. For an exactly solvable model system, even a zero order approximation is able to accurately reproduce complex nonadiabatic dynamics with quantum nuclear effects. The use of Bohmian trajectories adds interpretative value to the method and provides a numerically stable algorithm to avoid the calculation of the unstable quantum potential. Nonetheless, other kinds of trajectory-based statistical techniques could be used as 
well. In this respect, the use of time-dependent density functional theory to sample the electronic subspace in combination with Eq. (4) is currently under study.

We gratefully acknowledge John C. Tully and Xavier Oriols for useful conversations. G. A. acknowledges the Beatriu de Pinós Program for financial support through Project No. 2010BP-A00069. I. F. thanks the Alexander von Humboldt Foundation for financial support. A. R. acknowledges support by the European Research Council Advanced Grant DYNamo (No. ERC-2010AdG-267374), a Spanish Grant (No. FIS2010- 21282C02-01), Grupos Consolidados UPV/EHU del Gobierno Vasco (IT-578-13), and the Ikerbasque and European Commission Project CRONOS (Grant No. 280879- 2).

*albareda@fhi-berlin.mpg.de

†angel.rubio@ehu.es

[1] J. C. Tully, J. Chem. Phys. 137, 22A301 (2012).

[2] W. Domcke and G. Stock, in Advances in Chemical Physics (Wiley, New York, 2007), p. 1.

[3] M. Beck, A. Jäckle, G. Worth, and H.-D. Meyer, Phys. Rep. 324, 1 (2000).

[4] G. A. Worth and M. A. Robb, in The Role of Degenerate States in Chemistry (Wiley, New York, 2003), p. 355; D. Truhlar, in The Reaction Path in Chemistry: Current Approaches and Perspectives, Understanding Chemical Reactivity Vol. 16, edited by D. Heidrich (Springer, New York, 1995), p. 229.

[5] J. C. Tully, Faraday Discuss. 110, 407 (1998).

[6] S. Sawada and H. Metiu, J. Chem. Phys. 84, 227 (1986); I. Burghardt, H.-D. Meyer, and L. S. Cederbaum, J. Chem. Phys. 111, 2927 (1999); T. J. Martinez, M. Ben-Nun, and R. D. Levine, J. Phys. Chem. 100, 7884 (1996).

[7] A. Sanchez-Galvez, P. Hunt, M. A. Robb, M. Olivucci, T. Vreven, and H. B. Schlegel, J. Am. Chem. Soc. 122, 2911 (2000); F. Jolibois, M. J. Bearpark, S. Klein, M. Olivucci, and M. A. Robb, J. Am. Chem. Soc. 122, 5801 (2000); M. Ben-Nun, F. Molnar, H. Lu, J. C. Phillips, T. J. Martinez, and K. Schulten, Faraday Discuss. 110, 447 (1998).

[8] X. Oriols, Phys. Rev. Lett. 98, 066803 (2007).
[9] G. Albareda, J. Suñé, and X. Oriols, Phys. Rev. B 79, 075315 (2009); G. Albareda, D. Marian, A. Benali, S. Yaro, N. Zangh, and X. Oriols, J. Comput. Electron. 12, 405 (2013); G. Albareda, F. L. Traversa, A. Benali, and X. Oriols, Fluct. Noise Lett. 11, 1242008 (2012).

[10] X. Oriols and J. Mompart, Applied Bohmian Mechanics: From Nanoscale Systems to Cosmology (Pan Stanford, Singapore, 2012); P. Holland, The Quantum Theory of Motion: An Account of the de Broglie-Bohm Causal Interpretation of Quantum Mechanics (Cambridge University Press, Cambridge, England, 1995).

[11] A. Benseny, J. Bagudà, X. Oriols, and J. Mompart, Phys. Rev. A 85, 053619 (2012); J. Wu, B. B. Augstein, and C. Figueira de Morisson Faria, Phys. Rev. A 88, 023415 (2013); Y. Song, S.-Y. Li, X.-S. Liu, F.-M. Guo, and Y.-J. Yang, Phys. Rev. A 88, 053419 (2013); N. Takemoto and A. Becker, J. Chem. Phys. 134, 074309 (2011).

[12] I. Tavernelli, Phys. Rev. A 87, 042501 (2013); C. L. Lopreore and R. E. Wyatt, J. Chem. Phys. 116, 1228 (2002); E. Gindensperger, C. Meier, and J. A. Beswick, J. Chem. Phys. 113, 9369 (2000); O. V. Prezhdo and C. Brooksby, Phys. Rev. Lett. 86, 3215 (2001).

[13] A. Abedi, N. T. Maitra, and E. K. U. Gross, Phys. Rev. Lett. 105, 123002 (2010).

[14] See Appendix A of the Supplemental Material at http://link .aps.org/supplemental/10.1103/PhysRevLett.113.083003 for a detailed derivation of the connection between the conditional formalism and the exact factorization of the molecular wave function.

[15] See Appendix B of the Supplemental Material at http://link .aps.org/supplemental/10.1103/PhysRevLett.113.083003, which includes Refs. [16,17], for a detailed discussion of the performance of the Hermitian conditional scheme to describe the splitting of the nuclear wave packet.

[16] A. Abedi, F. Agostini, Y. Suzuki, and E. K. U. Gross, Phys. Rev. Lett. 110, 263001 (2013).

[17] J. C. Tully, J. Chem. Phys. 93, 1061 (1990).

[18] S. Shin and H. Metiu, J. Chem. Phys. 102, 9285 (1995).

[19] Whenever the advective and or kinetic correlation terms are not negligible, the solution of the equations of motion (4) and (5) on a finite difference grid usually becomes unstable and requires the use of nonstandard propagation methods.

[20] E. A. McCullough and R. E. Wyatt, J. Chem. Phys. 51, 1253 (1969); 54, 3578 (1971). 